\title{
Constructing Simultaneous Hecke Eigenforms
}

\author{
T. Shemanske, S. Treneer and L. Walling
}

\begin{abstract}
It is well known that newforms of integral weight are simultaneous eigenforms for all the Hecke operators, and that the converse is not true. In this paper, we give a characterization of all simultaneous Hecke eigenforms associated to a given newform, and provide several applications. These include determining the number of linearly independent simultaneous eigenforms in a fixed space which correspond to a given newform, and characterizing several situations in which the full space of cusp forms is spanned by a basis consisting of such eigenforms. Part of our results can be seen as a generalization of results of Choie-Kohnen who considered diagonalization of "bad" Hecke operators on spaces with square free level and trivial character. Of independent interest, but used herein, is a lower bound for the dimension of the space of newforms with arbitrary character.
\end{abstract}

\section{Introduction}

For $N$ a positive integer, $\psi$ a Dirichlet character defined modulo $N$, and $k \geq 2$ an integer, we let $S_{k}(N, \psi)$ denote the space of cusp forms of weight $k$ for $\Gamma_{0}(N)$ with character $\psi$, and $S_{k}^{+}(N, \psi)$ the subspace generated by the newforms. For a prime $p$, we let $T_{p}$ (or $T_{p}^{N}$ ) denote the $p$ th Hecke operator for forms on $S_{k}(N, \psi)$. We use this notation for the primes which divide the level as well, so for example if $q \mid N$, our Hecke operator $T_{q}^{N}$ is the same as the operator $U_{q}$ in the notation of $[\mathbf{3}]$.

It is well-known that $S_{k}(N, \psi)$ has a basis consisting of simultaneous eigenforms for the algebra of Hecke operators generated by $\left\{T_{p}^{N} \mid(p, N)=1\right\}$, and via multiplicity-one that $S_{k}^{+}(N, \psi)$ has a basis of simultaneous eigenforms for all the Hecke operators. Since $S_{k}^{+}(N, \psi)$ is generally a proper subspace of $S_{k}(N, \psi)$, it is a natural question to consider the extent to which the full space of cusp forms has a basis of simultaneous eigenforms for all the Hecke operators. Choie and Kohnen [2] considered the question of diagonalizing "bad" Hecke operators (that is, $T_{q}^{N}$ where $q \mid N$ ), and gave an upper bound for the number of primes $q$ for which $T_{q}^{N}$ could not be diagonalized on $S_{k}(N, \psi)$ where $N$ is square-free, $q \mid N$ and $\psi$ is trivial. An alternate perspective on that question is to determine conditions under which simultaneous Hecke eigenforms are newforms. One result along these lines is Li's [3] Theorem 9: if $f \in S_{k}(N, \psi)$ is a simultaneous eigenform for all Hecke operators $T_{p}^{N}$, and $f$ is also an eigenform for the operator $K W_{N}$ (where $K$ is the conjugation operator and $W_{N}$ is the Fricke involution), then $f$ is a newform.

In this paper we address the question broadly, in particular giving a characterization of all simultaneous Hecke eigenforms associated to a given newform for arbitrary level and character. For a given newform $h \in S_{k}\left(N_{0}, \psi\right)$, we first determine (Theorem 2.1 and Proposition 2.2) the exact structure and the eigenvalues of each form $f \in S_{k}(N, \psi)$ which is Hecke-equivalent to $h$ and also an eigenfunction for $T_{q}^{N}$. In section 3 , we address the diagonalizability of $T_{q}^{N}$ on a given space of cusp forms, characterizing several situations in which the full space of cusp forms 
is spanned by a basis consisting of such eigenforms, as well as those situations when it is not (Theorems 3.3 and 3.5) . To establish the later result we derive a lower bound (Theorem 6.1) for the dimension of the space of newforms, $S_{k}^{+}(N, \psi)$; dimension formulas for the space of newforms with trivial character are given by Martin [4]. In Theorem 3.4, we generalize the results of Choie-Kohnen producing an upper bound for the number of primes $q$ for which $T_{q}^{N}$ fails to diagonalize. Section 4 considers simultaneous Hecke eigenforms, and section 5 has several examples delineating cases in which bases of simultaneous eigenforms do or do not exist.

\section{Characterizing Hecke Eigenforms at primes dividing the level}

Throughout, we make the convention that all Dirichlet characters will be considered as defined modulo their conductor, so that when considering a modular form in $S_{k}(N, \psi), \psi(d) \neq 0$ iff $d$ is relatively prime to the conductor. In particular, there may well be primes $q \mid N$ for which $\psi(q) \neq 0$. Of course for any prime $q \nmid N, \psi(q) \neq 0$. The convention is necessary to allow a uniform handling of all subspaces $S_{k}\left(N_{0}, \psi\right)$ where cond $(\psi)\left|N_{0}\right| N$.

Let $h \in S_{k}\left(N_{0}, \psi\right)$ be a newform (always assumed nonzero), $N$ an integer divisible by $N_{0}$, and $f \in S_{k}(N, \psi)$ a nonzero simultaneous Hecke eigenform having the same eigenvalues as $h$ for all Hecke operators $T_{\ell}^{N}, \ell$ a prime with $(\ell, N)=1$. The eigenvalues of $h$ are given by $h \mid T_{\ell}^{N_{0}}=\lambda_{\ell} h$ for all primes $\ell$, and we note that $T_{\ell}^{N}=T_{\ell}^{N_{0}}$ when $(\ell, N)=1$. Moreover suppose that $f$ is also a nonzero eigenform for $T_{q}^{N}$ where $q$ is a fixed prime dividing $N$, and put $f \mid T_{q}^{N}=\kappa_{q} f$. It is well-known $[\mathbf{1}],[\mathbf{3}]$, that $f$ has the form

$$
f=\sum_{d \mid \frac{N}{N_{0}}} \alpha_{d} h \mid B_{d}
$$

where $B_{d}$ is the shift operator of $[\mathbf{3}]$, and the $\alpha_{d}$ are complex scalars.

ThEOREM 2.1. Let the notation be as above. Then assuming $q \mid N$ and $d \mid N / N_{0}$, we have:

(i) If $q \mid N_{0}$ then $\alpha_{d}=0$ if $q^{2} \mid d$.

(a) If $q \nmid N / N_{0}$, then $\kappa_{q}=\lambda_{q}$, and (vacuously) $\alpha_{d}=0$ for $q \mid d$.

(b) If $q \mid N / N_{0}$, then $\alpha_{d}=\left(\kappa_{q}-\lambda_{q}\right) \alpha_{d / q}$ if $q \| d$. If $\kappa_{q} \neq 0$, then $\kappa_{q}=\lambda_{q}$, and $\lambda_{q}=0$ implies $\kappa_{q}=0$.

(ii) If $q \nmid N_{0}$, then $\alpha_{d}=0$ if $q^{3} \mid d$.

(a) If $\kappa_{q} \neq 0$, then $\alpha_{d}=0$ if $q^{2} \mid d, \quad \alpha_{d}=\left(\kappa_{q}-\lambda_{q}\right) \alpha_{d / q}$ if $q \| d$, and $\kappa_{q}=$ $\frac{1}{2}\left(\lambda_{q} \pm \sqrt{\lambda_{q}^{2}-4 \psi(q) q^{k-1}}\right) \neq \lambda_{q}$.

(b) If $\kappa_{q}=0$, then $q^{2} \mid N / N_{0}, \alpha_{d}=\psi(q) q^{k-1} \alpha_{d / q^{2}}$ if $q^{2} \| d$, and $\alpha_{d}=-\lambda_{q} \alpha_{d / q}$ if $q \| d$.

Proof. As above, we assume that $f=\sum_{d \mid \frac{N}{N_{0}}} \alpha_{d} h \mid B_{d}$. We separate the argument by cases.

- Case: $q \mid N_{0}, q \nmid N / N_{0}$.

Since $q \mid N_{0}, T_{q}^{N}=T_{q}^{d N_{0}}$ for any $d$. Also note that since $q \nmid N / N_{0}$, any divisor $d \mid N / N_{0}$ satisfies $(d, q)=1$, so that the shift and Hecke operators commute: $B_{d}\left|T_{q}^{d N_{0}}=T_{q}^{N_{0}}\right| B_{d}$. Thus

$$
\kappa_{q} f=f\left|T_{q}^{N}=\sum_{d \mid \frac{N}{N_{0}}} \alpha_{d} h\right| B_{d}\left|T_{q}^{d N_{0}}=\sum_{d \mid \frac{N}{N_{0}}} \alpha_{d} h\right| T_{q}^{N_{0}}\left|B_{d}=\lambda_{q} \sum_{d \mid \frac{N}{N_{0}}} \alpha_{d} h\right| B_{d}=\lambda_{q} f,
$$

and so we have $\kappa_{q}=\lambda_{q}$. Since $q \nmid N / N_{0}$, it is vacuously true that $\alpha_{d}=0$ for $q \mid d$.

- Case: $q\left|N_{0}, q\right| N / N_{0}$. 
As in the previous case, we note that $q \mid N_{0}$ implies $T_{q}^{N}=T_{q}^{d N_{0}}$ for any $d$, and for any divisor $d \mid N / N_{0}$ satisfying $(d, q)=1$, the shift and Hecke operators commute: $B_{d} \mid T_{q}^{d N_{0}}=$ $T_{q}^{N_{0}} \mid B_{d}$. Finally we note that $B_{q} T_{q}^{N}=1$. With these observations we have

$$
\begin{aligned}
\kappa_{q} f=f \mid T_{q}^{N} & =\sum_{d \mid N / N_{0}} \alpha_{d} h\left|B_{d}\right| T_{q}^{d N_{0}} \\
& =\sum_{d, q \nmid d} \alpha_{d} h\left|T_{q}^{N_{0}}\right| B_{d}+\sum_{d, q \mid d} \alpha_{d} h \mid B_{d / q} \\
& =\sum_{d, q \nmid d}\left(\lambda_{q} \alpha_{d}+\alpha_{d q}\right) h\left|B_{d}+\sum_{d, q^{2} \mid d} \alpha_{d} h\right| B_{d / q} .
\end{aligned}
$$

We now show the second summand does not appear.

Lemma. $\quad \alpha_{d}=0$ if $q^{2} \mid d$.

Proof. If $\kappa_{q}=0$, then the linear independence of $\left\{h \mid B_{d}\right\}$ yields the result. If $\kappa_{q} \neq 0$, let $M=\max _{d \mid N / N_{0}}\left\{\operatorname{ord}_{q}(d) \mid \alpha_{d} \neq 0\right\}$. Then we have

$$
\begin{aligned}
\kappa_{q} f & =\sum_{d \mid N / N_{0}} \kappa_{q} \alpha_{d} h\left|B_{d}=\sum_{i=0}^{M} \sum_{d \mid N / N_{0}, q^{i} \| d} \kappa_{q} \alpha_{d} h\right| B_{d} \\
& =\sum_{d, q \nmid d}\left(\lambda_{q} \alpha_{d}+\alpha_{d q}\right) h\left|B_{d}+\sum_{d, q^{2} \mid d} \alpha_{d} h\right| B_{d / q} .
\end{aligned}
$$

There is no issue if $M<2$, so we assume $M \geq 2$. In that case for a divisor $d$ with $\operatorname{ord}_{q}(d)=M$, we see that a term with $h \mid B_{d}$ occurs as a summand in $\kappa_{q} f$, but not in $\sum_{d, q^{2} \mid d} \alpha_{d} h \mid B_{d / q}$, so $\alpha_{d}=0$, a contradiction.

Applying this observation, the equation above becomes:

$$
\sum_{d \mid N / N_{0}, q^{2 \nmid d}} \kappa_{q} \alpha_{d} h\left|B_{d}=\kappa_{q} f=f\right| T_{q}^{N}=\sum_{d \mid N / N_{0}, q \nmid d}\left(\lambda_{q} \alpha_{d}+\alpha_{d q}\right) h \mid B_{d} .
$$

By the linear independence of the set $\left\{h \mid B_{d}\right\}$, we deduce from Equation 2.1 that $\kappa_{q} \alpha_{d}=0$ when $q \| d$. If $\kappa_{q}=0$, then Equation 2.1 is zero, hence the coefficients of $h \mid B_{d}$ are all zero and we conclude

$$
\alpha_{d}=-\lambda_{q} \alpha_{d / q}=\left(\kappa_{q}-\lambda_{q}\right) \alpha_{d / q} \quad \text { if } q \| d .
$$

On the other hand, if $\kappa_{q} \neq 0$, then $\alpha_{d}=0$ when $q \| d$, so Equation 2.1 becomes

$$
\kappa_{q} f=\sum_{d \mid N / N_{0}, q \nmid d} \kappa_{q} \alpha_{d} h\left|B_{d}=\sum_{d \mid N / N_{0}, q \nmid d} \lambda_{q} \alpha_{d} h\right| B_{d}=\lambda_{q} f
$$

and hence $\kappa_{q}=\lambda_{q}$. It follows that $\lambda_{q}=0$ implies $\kappa_{q}=0$, and $0=\alpha_{d}=\left(\kappa_{q}-\lambda_{q}\right) \alpha_{d / q}$ if $q \| d$.

- Case: $q \nmid N_{0}, q \mid N / N_{0}$. 
In this case $T_{q}^{N}=T_{q}^{N_{0}}-\psi(q) q^{k-1} B_{q}$, and we have

$$
\begin{aligned}
\kappa_{q} f & =\sum_{d \mid N / N_{0}} \kappa_{q} \alpha_{d} h\left|B_{d}=\sum_{d \mid N / N_{0}} \alpha_{d} h\right| B_{d} \mid T_{q}^{N} \\
& =\sum_{d, q \nmid d} \alpha_{d} h\left|T_{q}^{N}\right| B_{d}+\sum_{d, q \mid d} \alpha_{d} h \mid B_{d / q} \\
& =\sum_{d, q \nmid d} \alpha_{d} h\left|\left(T_{q}^{N_{0}}-\psi(q) q^{k-1} B_{q}\right)\right| B_{d}+\sum_{d, q \mid d} \alpha_{d} h \mid B_{d / q} \\
& =\sum_{d, q \nmid d}\left(\lambda_{q} \alpha_{d}+\alpha_{d q}\right) h\left|B_{d}+\sum_{d, q \| d}\left(\alpha_{d q}-\psi(q) q^{k-1} \alpha_{d / q}\right) h\right| B_{d}+\sum_{d, q^{3} \mid d} \alpha_{d} h \mid B_{d / q} .
\end{aligned}
$$

To simplify this expression, we show

LEMMA. $\quad \alpha_{d}=0$ if $q^{3} \mid d$.

Proof. This is completely analogous to the previous lemma. Let $M=\max _{d \mid N / N_{0}}\left\{\operatorname{ord}_{q}(d) \mid\right.$ $\left.\alpha_{d} \neq 0\right\}$. There is no issue if $M<3$, so assume $M \geq 3$. In that case for a divisor $d$ with $\operatorname{ord}_{q}(d)=M$, we see that a term with $h \mid B_{d}$ occurs in $\kappa_{q} f$, but not $\sum_{d, q^{3} \mid d} \alpha_{d} h \mid B_{d / q}$, so $\alpha_{d}=0$, a contradiction.

To go further, we first suppose that $\kappa_{q} \neq 0$. If $q \| N / N_{0}$, we have $\alpha_{d}=0$ for $q^{2} \mid d$ by convention. Otherwise, let $d \mid N / N_{0}$ with $q^{2} \mid d$. The coefficient of $h \mid B_{d}$ in $\kappa_{q} f$ is $\kappa_{q} \alpha_{d}$ while it is $\alpha_{d q}$ in $\sum_{d, q^{3} \mid d} \alpha_{d} h \mid B_{d / q}$. By the lemma, $\alpha_{q d}=0$, so we infer $\alpha_{d}=0$.

Applying these observations to the above expression for $\kappa_{q} f$ yields

$$
\kappa_{q} f=\sum_{d \mid N / N_{0}} \kappa_{q} \alpha_{d} h\left|B_{d}=\sum_{d \mid N / N_{0}, q \nmid d}\left(\lambda_{q} \alpha_{d}+\alpha_{d q}\right) h\right| B_{d}+\sum_{d \mid N / N_{0}, q \nmid d}-\psi(q) q^{k-1} \alpha_{d} h \mid B_{d q} .
$$

Comparing coefficients of $h \mid B_{d}$ and $h \mid B_{d q}$ we obtain for $q \| d$ :

$$
\begin{aligned}
\alpha_{d} & =\left(\kappa_{q}-\lambda_{q}\right) \alpha_{d / q}, \text { and } \\
\kappa_{q} \alpha_{d} & =-\psi(q) q^{k-1} \alpha_{d / q} .
\end{aligned}
$$

Substituting the expression for $\alpha_{d}$ from the first equation into the second yields the quadratic $\left(\kappa_{q}^{2}-\lambda_{q} \kappa_{q}+\psi(q) q^{k-1}\right) \alpha_{d / q}=0$. Note that $\alpha_{d / q}=0$ for all $d$ with $q \| d$ would imply $\alpha_{d}=0$ for all $d \mid N / N_{0}$, hence $f=0$. Thus

$$
\kappa_{q}=\frac{1}{2}\left(\lambda_{q} \pm \sqrt{\lambda_{q}^{2}-4 \psi(q) q^{k-1}}\right),
$$

and we note that $\kappa_{q} \neq \lambda_{q}$ since $\psi(q) \neq 0$.

Finally, we assume $\kappa_{q}=0$. Then all the coefficients of the $h \mid B_{d}$ in equation 2.2 are zero, yielding $\alpha_{d} \lambda_{q}+\alpha_{d q}=0$ for $q \nmid d$, and $\alpha_{d q}=\psi(q) q^{k-1} \alpha_{d / q}$ for $q \| d$. Note that if $q \| N / N_{0}$, by convention we would have $\alpha_{d q}=0$ in the last equation, leading to $\alpha_{d / q}=0$ and hence $\alpha_{d}=0$ implying $f=0$. Thus $\kappa_{q}=0$ forces $q^{2} \mid N / N_{0}$, which completes the proof.

As above, let $h \in S_{k}\left(N_{0}, \psi\right)$ be a newform, and $N$ an integer divisible by $N_{0}$. Denote the class of $h$ by

$$
[h]=\left\{f \in S_{k}(N, \psi): f\left|T_{p}^{N}=h\right| T_{p}^{N} \text { for all primes } p \nmid N\right\} .
$$


By the theory of newforms, we know

$$
[h]=\bigoplus_{d \mid N / N_{0}}\left\langle h \mid B_{d}\right\rangle,
$$

that is, $f \in[h]$ if and only if $f=\sum_{d \mid N / N_{0}} \alpha_{d} h \mid B_{d}$ for scalars $\alpha_{d}$. It is clear from the general theory of newforms that any such $f$ is a simultaneous eigenform for all Hecke operators $T_{p}^{N}$ for primes $p \nmid N$. In Theorem 2.1, we have given necessary conditions on the coefficients $\alpha_{d}$ for $f$ to be an eigenform for $T_{q}^{N}$ for a prime $q \mid N$ and eigenvalue $\kappa_{q}$. However, the necessary conditions are also sufficient.

Proposition 2.2. Let $h \in S_{k}\left(N_{0}, \psi\right)$ be a newform, $N$ an integer divisible by $N_{0}$, and $q$ a prime dividing $N$. Set $h \mid T_{q}^{N_{0}}=\lambda_{q} h$, and fix $\kappa_{q}$ and constants $\alpha_{d}$ for $d \mid N / N_{0}$ according to the following scheme (any unconstrained constants are arbitrary):

- $q \mid N_{0}$ and $q \nmid N / N_{0}:$ Let $\kappa_{q}=\lambda_{q}$.

$-q \mid N_{0}$ and $q \mid N / N_{0}$ : Let $\kappa_{q}=\lambda_{q}$ or 0 , and put $\alpha_{d}=0$ if $q^{2} \mid d$, and $\alpha_{d}=\left(\kappa_{q}-\lambda_{q}\right) \alpha_{d / q}$ if $q \| d$.

$-q \nmid N_{0}$ : Set $\alpha_{d}=0$ if $q^{3} \mid d$.

* Let $\kappa_{q}=\frac{1}{2}\left(\lambda_{q} \pm \sqrt{\lambda_{q}^{2}-4 \psi(q) q^{k-1}}\right)$, and note $\kappa_{q} \neq 0, \lambda_{q}$. For $q^{2} \mid d$ put $\alpha_{d}=0$; for $q \| d$, put $\alpha_{d}=\left(\kappa_{q}-\lambda_{q}\right) \alpha_{d / q}$.

* Moreover, if $q^{2} \mid N$, we can also let $\kappa_{q}=0$, and for $q^{2} \| d$, put $\alpha_{d}=\psi(q) q^{k-1} \alpha_{d / q^{2}}$ and for $q \| d$, put $\alpha_{d}=-\lambda_{q} \alpha_{d / q}=\left(\kappa_{q}-\lambda_{q}\right) \alpha_{d / q}$.

Then $f=\sum_{d \mid N / N_{0}} \alpha_{d} h \mid B_{d}$ is an eigenform for $T_{q}^{N}$ with eigenvalue $\kappa_{q}$.

Proof. The proposition follows immediately from the computations already present in Theorem 2.1.

\section{Comparison to Choie-Kohnen}

As in the previous section, let $h \in S_{k}\left(N_{0}, \psi\right)$ be a newform, and $N$ an integer divisible by $N_{0}$, and denote the class of $h$ by $[h]$. From $[\mathbf{1}],[\mathbf{3}]$ we know that if $S_{k}^{+}\left(N_{0}, \psi\right)$ denotes the space generated by newforms of level $N_{0}$,

$$
\begin{aligned}
S_{k}(N, \psi) & =\bigoplus_{\operatorname{cond}(\psi)\left|N_{0}\right| N} \bigoplus_{d \mid N / N_{0}}\left(S_{k}^{+}\left(N_{0}, \psi\right) \mid B_{d}\right) \\
& =\bigoplus_{\operatorname{cond}(\psi)\left|N_{0}\right| N} \bigoplus_{h}[h],
\end{aligned}
$$

where the last sum is over normalized newforms $h \in S_{k}^{+}\left(N_{0}, \psi\right)$.

LEMma 3.1. $T_{q}^{N}$ is diagonalizable on $S_{k}(N, \psi)$ if and only if there is a basis of $S_{k}(N, \psi)$ consisting of simultaneous eigenforms for $T_{q}^{N}$ as well as for all $T_{p}^{N}, p$ a prime with $p \nmid N$. Moreover, for each $N_{0} \mid N$ and each normalized newform $h \in S_{k}\left(N_{0}, \psi\right), T_{q}^{N}$ is diagonalizable on $S_{k}(N, \psi)$ if and only if it is diagonalizable on each class $[h]$.

Proof. For both statements, only the forward direction requires proof. If $T_{q}^{N}$ is diagonalizable on $S_{k}(N, \psi)$, then $S_{k}(N, \psi)=\bigoplus_{i} E_{i}$ where the $E_{i}$ are the eigenspaces corresponding to 
the distinct eigenvalues of $T_{q}^{N}$. For a prime $p \nmid N$, the Hecke operators $T_{p}^{N}$ and $T_{q}^{N}$ commute so each eigenspace is invariant under all the $T_{p}^{N}, p \nmid N$. Since Hecke theory tells us that $S_{k}(N, \psi)$ admits a basis of simultaneous eigenforms for all the $T_{p}^{N}$, and each $E_{i}$ is invariant under this collection of operators, each $E_{i}$ also admits such a basis, $\mathcal{B}_{i}$, every element of which is also (by definition) an eigenform for $T_{q}^{N}$.

Now consider the second statement. Every element of the basis $\mathcal{B}_{i}$ belongs to a unique class $[h]$ of some newform $h \in S_{k}\left(N_{0}, \psi\right)$ with $N_{0} \mid N$. We collect the elements of the $\mathcal{B}_{i}$ which belong to a given class $[h]$. Since $S_{k}(N, \psi)$ is the direct sum of such classes and all the $\mathcal{B}_{i}$ taken together span $S_{k}(N, \psi)$, we see that $T_{q}^{N}$ is diagonalizable on each class $[h]$.

Below we reverse the process of the lemma, starting with the class of a newform $[h]$, and investigate how to decompose the class $[h]$ into subspaces, extracting the various eigenspaces of $T_{q}^{N}$ for $q \mid N$, and give conditions under which $T_{q}^{N}$ can be diagonalized on $[h]$. We then use these results to generalize those of Choie and Kohnen [2]. We also apply these results in section 4 to determine when there exist simultaneous eigenforms for all the Hecke operators, and determine the number of such eigenforms which are linearly independent.

For a prime $q \mid N$ and $h \in S_{k}\left(N_{0}, \psi\right)$ a newform, Theorem 2.1 implies that [h] contains at most three eigenspaces for $T_{q}^{N}$. With $f \mid T_{q}^{N}=\kappa_{q} f$, we have

$$
\kappa_{q}= \begin{cases}\lambda_{q} & \text { when } q \nmid N / N_{0}, \\ 0, \lambda_{q} & \text { when } q\left|N / N_{0}, q\right| N_{0}, \\ \frac{1}{2}\left(\lambda_{q} \pm \sqrt{\lambda_{q}^{2}-4 \psi(q) q^{k-1}}\right) & \text { when } q \| N / N_{0}, q \nmid N_{0}, \text { and } \\ 0, \frac{1}{2}\left(\lambda_{q} \pm \sqrt{\lambda_{q}^{2}-4 \psi(q) q^{k-1}}\right) & \text { when } q^{2} \mid N / N_{0}, q \nmid N_{0} .\end{cases}
$$

When $q \nmid N / N_{0}$, we have observed that every element of [h] is an eigenform for $T_{q}^{N}$ having eigenvalue $\lambda_{q}$, so $T_{q}^{N}$ diagonalizes on $[h]$. Thus we restrict our attention to the case where $q \mid N / N_{0}$. Write $N / N_{0}=q^{\mu} M_{0}$, with $q \nmid M_{0}$. For $d_{0} \mid M_{0}$, put $U_{d_{0}}=\bigoplus_{i=0}^{\mu}\left\langle h \mid B_{d_{0} q^{i}}\right\rangle$ where $\left\langle h \mid B_{d}\right\rangle$ denotes the $\mathbb{C}$-linear span of $h \mid B_{d}$. Using that $[h]=\bigoplus_{d_{0} \mid M_{0}} U_{d_{0}}$, Theorem 2.1 shows that every eigenform $f \in[h]$ with $f \mid T_{q}^{n}=\kappa_{q} f$ has the form $f=\sum_{d_{0} \mid M_{0}} f_{d_{0}}$ with $f_{d_{0}} \in U_{d_{0}}$, and Proposition 2.2 shows that each $f_{d_{0}}$ also satisfies $f_{d_{0}} \mid T_{q}^{N}=\kappa_{q} f_{d_{0}}$. Thus $T_{q}^{N}$ diagonalizes on $[h]$ if and only if it diagonalizes on each $U_{d_{0}}$. Further, Theorem 2.1 and Proposition 2.2 also show that each subspace $U_{d_{0}}$ contains precisely $m$ linearly independent eigenforms for $T_{q}^{N}$ where $m$ is the number of distinct eigenvalues $\kappa_{q}$ given in Equation 3.1. Since the dimension of $U_{d_{0}}=\mu+1, T_{q}^{N}$ diagonalizes on $[h]$ if and only if $m=\mu+1$. Note that since $m \leq 3, T_{q}^{N}$ diagonalizes on $[h]$ only if $\mu \leq 2$. Moreover when $\mu=2$ and $q \mid N_{0}$, we see from above that there are most $m=2<3=\mu+1$ distinct eigenvalues, so once again $T_{q}^{N}$ cannot diagonalize in this case.

We quantify the above observations a bit further. Still assuming $q \mid N / N_{0}$, if $q \mid N_{0}$, there are two distinct eigenvalues precisely when $\lambda_{q} \neq 0$; by Theorem 3 of $[\mathbf{3}]$ this occurs iff $q \| N_{0}$ or $\operatorname{ord}_{q}(\operatorname{cond}(\psi))=\operatorname{ord}_{q}\left(N_{0}\right)$. If $q \nmid N_{0}$, there are two independent eigenforms for $T_{q}^{N}$ (with non-zero eigenvalues $\kappa_{q}$ ) precisely when $\lambda_{q}^{2} \neq 4 \psi(q) q^{k-1}$, that is when $\lambda_{q}$ fails to achieve the Deligne bound. There is an additional independent eigenform with eigenvalue $\kappa_{q}=0$ if and only if $\mu \geq 2$. For later convenience we denote by $\mathfrak{Q}_{N_{0}, h}$ the set of primes $q \mid N / N_{0}$ (just characterized) yielding a maximal number of distinct eigenvalues $\kappa_{q}$, and tabulate their number.

\begin{tabular}{|c|c||c|}
\hline \multicolumn{2}{|c||}{$\mu=\operatorname{ord}_{q}\left(N / N_{0}\right) \geq 1 ; q \in \mathfrak{Q}_{N_{0}, h}$ provided: } & Number of distinct eigenvalues $\kappa_{q}$ \\
\hline$q \mid N_{0}$ & $\operatorname{ord}_{q}(\operatorname{cond}(\psi))=\operatorname{ord}_{q}\left(N_{0}\right)$ or $q \| N_{0}$ & 2 \\
\hline$q \nmid N_{0}$ & $\lambda_{q}^{2} \neq 4 \psi(q) q^{k-1}$ & $\min (3, \mu+1)$ \\
\hline
\end{tabular}




\begin{tabular}{|l|c||c|}
\hline \multicolumn{2}{|c||}{$\mu=\operatorname{ord}_{q}\left(N / N_{0}\right) \geq 1 ; q \notin \mathfrak{Q}_{N_{0}, h}$ provided: } & Number of distinct eigenvalues $\kappa_{q}$ \\
\hline$q \mid N_{0}$ & $q^{2} \mid N_{0}$ and $q \mid N_{0} / \operatorname{cond}(\psi)$ & 1 \\
\hline$q \nmid N_{0}$ & $\lambda_{q}^{2}=4 \psi(q) q^{k-1}$ & $\min (2, \mu)$ \\
\hline
\end{tabular}

With this in hand, we now generalize the first part of Choie and Kohnen's theorem [2] characterizing when "bad" Hecke operators can be diagonalized.

Theorem 3.2. For a prime $q \mid N$, the Hecke operator $T_{q}^{N}$ is diagonalizable on $S_{k}(N, \psi)$ only if $S_{k}(N, \psi)$ contains no newform of level $N_{0}$ with $q^{3} \mid N / N_{0}$, or with $q^{2} \mid N / N_{0}$ and $q \mid N_{0}$. Assuming this condition, $T_{q}^{N}$ is diagonalizable if and only if for each $N_{0}$ with $\operatorname{cond}(\psi)\left|N_{0}\right| N$ and each newform $h \in S_{k}\left(N_{0}, \psi\right)$ with $h \mid T_{q}^{N_{0}}=\lambda_{q} h$, either $q \nmid N / N_{0}$ or $q \in \mathfrak{Q}_{N_{0}, h}$.

Proof. We know that

$$
S_{k}(N, \psi)=\bigoplus_{\operatorname{cond}(\psi)\left|N_{0}\right| N} \bigoplus_{h}[h],
$$

where the sum is over normalized newforms $h \in S_{k}\left(N_{0}, \psi\right)$. By Lemma 3.1, it suffices to determine when $T_{q}^{N}$ is diagonalizable on each class [h]. Given a newform $h \in S_{k}\left(N_{0}, \psi\right)$, we have seen from the discussion preceding the theorem that $T_{q}^{N}$ is diagonalizable on $[h]$ only if $\mu=\operatorname{ord}_{q}\left(N / N_{0}\right) \leq 2$ and if $\mu=2, q \nmid N_{0}$. Thus the given conditions are necessary. Moreover, if $q \nmid N / N_{0}$, every element of $[h]$ is an eigenform for $T_{q}^{N}$, so we restrict our attention to the case $q \mid N / N_{0}$.

Consider a newform $h \in S_{k}\left(N_{0}, \psi\right)$. As before, write $N / N_{0}=q^{\mu} M_{0}$, with $q \nmid M_{0}$, and recall we are assuming $\mu=1$ or $\mu=2$ and $q \nmid N_{0}$. For $d_{0} \mid M_{0}$, put $U_{d_{0}}=\bigoplus_{i=0}^{\mu}\left\langle h \mid B_{d_{0} q^{i}}\right\rangle$. We have observed above since $[h]=\bigoplus_{d_{0} \mid M_{0}} U_{d_{0}}$, that $T_{q}^{N}$ diagonalizes on $[h]$ if and only if it diagonalizes on each $U_{d_{0}}$, and that $T_{q}^{N}$ diagonalizes on $U_{d_{0}}$ if and only if $\operatorname{dim} U_{d_{0}}$ is equal to the number of distinct eigenvalues $\kappa_{q}$. From the tables above it is clear that the dimension $(\mu+1)$ equals the number of distinct eigenvalues if and only if $q \in \mathfrak{Q}_{N_{0}, h}$.

We summarize the above results in a more compact formulation.

Theorem 3.3. Let $q$ be prime, and let $\psi$ be a Dirichlet character with conductor $\mathfrak{f}=q^{\nu} M_{0}$, with $\nu \geq 0$ and $q \nmid M_{0}$. Let $M$ be an integer with $M_{0} \mid M$ and $q \nmid M$. If $s \leq 2$, then $T_{q}$ is diagonalizable on $S_{k}\left(q^{\nu+s} M, \psi\right)$ if and only if one of the following is true:

(i) $s=0$,

(ii) $s=1$ and $\nu \geq 1$,

(iii) $s>0, \nu=0$, and $S_{k}\left(q^{\nu+s} M, \psi\right)$ contains no newform $h$ of level $N_{0}$ with $q \nmid N_{0}, T_{q}^{N_{0}} h=$ $\lambda_{q} h$, and $\lambda_{q}^{2}=4 \psi(q) q^{k-1}$, or

(iv) $s=2, \nu \geq 1$, and $S_{k}\left(q^{\nu+s} M, \psi\right)$ contains no newform of level $N_{0}$ with $\operatorname{ord}_{q}\left(N_{0}\right)=\nu$ or $\nu+$ 1.

Proof. Set $N=q^{\nu+s} M$. We first interpret Theorem 3.2 in this setting. Since $s \leq 2, q^{3} \nmid N / \mathfrak{f}$, so $S_{k}(N, \psi)$ contains no newform of level $N_{0}$ with $q^{3} \mid N / N_{0}$. The only case in which $\mathfrak{f}\left|N_{0}\right| N$ with $q \mid N_{0}$ and $q^{2} \mid N / N_{0}$ occurs when $s=2$ and $\operatorname{ord}_{q}\left(N_{0}\right)=\nu \geq 1$. In this case, if $S_{k}(N, \psi)$ contains a newform of level $N_{0}$ then $T_{q}^{N}$ is not diagonalizable. Otherwise, $T_{q}^{N}$ is diagonalizable if and only if for each $\mathfrak{f}\left|N_{0}\right| N$ and each newform $h \in S_{k}\left(N_{0}, \psi\right)$, either $q \nmid N / N_{0}$ or $q \in \mathfrak{Q}_{N_{0}, h}$. 
First suppose that one of conditions (1) - (4) holds. If (1) holds then $q \nmid N / \mathfrak{f}$, so $q \nmid N / N_{0}$ for all $\mathfrak{f}\left|N_{0}\right| N$, hence $T_{q}^{N}$ is diagonalizable. If (2) holds, then $\operatorname{ord}_{q}\left(N_{0}\right)=\nu$ or $\nu+1$ for each $\mathfrak{f}\left|N_{0}\right| N$. In the first case, $\operatorname{ord}_{q}\left(N_{0}\right)=\operatorname{ord}_{q}(\mathfrak{f})$ so $q \in \mathfrak{Q}_{N_{0}, h}$ for each $h$ with such level. In the second case, $q \nmid N / N_{0}$. Therefore $T_{q}^{N}$ is diagonalizable. Now suppose that (3) holds. Then $q \in \mathfrak{Q}_{N_{0}, h}$ for each newform $h$ of level $N_{0}$ with $q \nmid N_{0}$. If $\operatorname{ord}_{q}\left(N_{0}\right)=s$ then $q \nmid N / N_{0}$. Finally if $s=2$ and $q \| N_{0}$, then $q \in \mathfrak{Q}_{N_{0}, h}$ for each $h$ with such level. Hence $T_{q}^{N}$ is diagonalizable. Lastly, suppose that (4) holds. Then each newform $h$ contained in $S_{k}(N, \psi)$ has level $N_{0}$ with $\operatorname{ord}_{q}\left(N_{0}\right)=\nu+2$, so that $q \nmid N / N_{0}$. Therefore $T_{q}^{N}$ is diagonalizable.

Now suppose that none of (1) through (4) is true. Then since (1) is false, $s=1$ or 2 . If $s=1$, then since (2) is false, $\nu=0$. Then since (3) is false, $S_{k}(N, \psi)$ must contain some newform $h$ of level $N_{0}$ with $q \nmid N_{0}$ and $T_{q}^{N_{0}} h=\lambda_{q} h$ with $\lambda_{q}^{2}=4 \psi(q) q^{k-1}$. Then $q \notin \mathfrak{Q}_{N_{0}, h}$, so $T_{q}^{N}$ is not diagonalizable. Now suppose that $s=2$. If $\nu=0$ then by the previous argument, $T_{q}^{N}$ is not diagonalizable. If $\nu \geq 1$ then since (4) is false, $S_{k}(N, \psi)$ must contain some newform $h$ of level $N_{0}$ with either $\operatorname{ord}_{q}\left(N_{0}\right)=\nu$ or $\nu+1$. If $\operatorname{ord}_{q}\left(N_{0}\right)=\nu$, then $q \mid N_{0}$ and $q^{2} \mid N / N_{0}$ so $T_{q}^{N}$ is not diagonalizable. If $\operatorname{ord}_{q}\left(N_{0}\right)=\nu+1$ then $\mathfrak{f} \mid N_{0} / q$ and $q^{2} \mid N_{0}$, so $q \notin \mathfrak{Q}_{N_{0}, h}$, and hence $T_{q}^{N}$ is not diagonalizable.

In the next result, we extend the work of Choie and Kohnen [2] (where they considered square-free level and trivial character) by showing that if $k$ is even, $s=1$ or 2 and $\nu=0$, then case (3) of Theorem 3.3 holds for all but finitely many primes $q$.

Theorem 3.4. Let $k$ be an even integer, and let $\psi$ be a Dirichlet character whose conductor $\mathfrak{f}$ divides $M$. Then $T_{q}$ is diagonalizable on both $S_{k}(q M, \psi)$ and $S_{k}\left(q^{2} M, \psi\right)$ for all primes $q \nmid M$ except for a finite number $r \leq C(M, k, \psi)$ of exceptions, where

$$
C(M, k, \psi):=\sum_{\operatorname{cond}(\psi)\left|M_{0}\right| M} \operatorname{dim} S_{k}^{+}\left(M_{0}, \psi\right)\left(1+\sum_{\mu \geq 1}\left[\frac{g_{M_{0}, k}}{2^{\mu}}\right]\right),
$$

and

$$
g_{M_{0}, k}=\sum_{\chi \bmod M_{0}} \operatorname{dim} S_{k}^{+}\left(M_{0}, \chi\right)
$$

Proof. By Theorem 3.3, the only way that a given $T_{q}$ can fail to diagonalize on either $S_{k}(q M, \psi)$ or $S_{k}\left(q^{2} M, \psi\right)$ is if there is a newform $h \in S_{k}\left(M_{0}, \psi\right)$ for some $M_{0}$ with $\mathfrak{f}\left|M_{0}\right| M$ which has $T_{q}^{M_{0}} h=\lambda_{q} h$ with $\lambda_{q}^{2}=4 \psi(q) q^{k-1}$. Fix an $M_{0}$ with $\mathfrak{f}\left|M_{0}\right| M$ and a newform $h \in$ $S_{k}\left(M_{0}, \psi\right)$ with eigenvalues $\lambda_{n}$. Let $K_{h}$ be the field obtained by adjoining all the $\lambda_{n}$ to $\mathbb{Q}$. It is known (Proposition 2.8 of $[\mathbf{6}],[\mathbf{7}]$ ) that $K_{h}$ is a number field and contains the $N$ th roots of unity which arise as values of $\psi$. Let $\zeta$ be a primitive $2 N$ th root of unity, so that $\mathbb{Q}\left(\zeta^{2}\right) \subset K_{h}$ and hence $K_{h}(\zeta) / K_{h}$ is at most a quadratic extension. Since $k$ is even, $\sqrt{q} \in K_{h}(\zeta)$ for each prime $q \nmid M$ such that $\lambda_{q}^{2}=4 \psi(q) q^{k-1}$. We call such a $q$ an exceptional prime for $h$. Now if $p_{1}, p_{2}, \ldots, p_{s}$ are different primes, the degree of $\mathbb{Q}\left(\sqrt{p_{1}}, \sqrt{p_{2}}, \ldots, \sqrt{p_{s}}\right) / \mathbb{Q}$ is $2^{s}$. Since

$$
\mathbb{Q} \subseteq \mathbb{Q}(\sqrt{q}: q \text { an exceptional prime for } h) \subseteq K_{h}(\zeta)
$$

and $K_{h}$ is a finite extension of $\mathbb{Q}$, there must be a finite number $r_{h}$ of exceptional primes for $h$. In particular, $r_{h} \leq \operatorname{ord}_{2}\left(\left[K_{h}: \mathbb{Q}\right]\right)+1$.

The group $\operatorname{Gal}(\overline{\mathbb{Q}} / \mathbb{Q})$ acts on normalized eigenforms in $S_{k}\left(M_{0}, \psi\right)$ by sending $f=\sum a(n) q^{n} \in$ $S_{k}\left(M_{0}, \psi\right)$ to $f^{\sigma}=\sum a(n)^{\sigma} q^{n} \in S_{k}\left(M_{0}, \psi^{\sigma}\right)$, for each $\sigma \in \operatorname{Gal}(\overline{\mathbb{Q}} / \mathbb{Q})$ (Proposition 2.6 of $[6]$, [7]). Let $K_{M_{0}, k}=\prod K_{h}$ be the composite field where the product runs over all characters $\chi$ modulo $M_{0}$ and all newforms $h \in S_{k}\left(M_{0}, \chi\right)$. Since each automorphism of the Galois closure of 
$K_{M_{0}, k} / \mathbb{Q}$ permutes these newforms, it can be considered as a subgroup of $S_{g_{M_{0}, k}}$, the symmetric group on $g_{M_{0}, k}$ elements, where

$$
g_{M_{0}, k}=\sum_{\chi \bmod M_{0}} \operatorname{dim} S_{k}^{+}\left(M_{0}, \chi\right) .
$$

Then $\left[K_{h}: \mathbb{Q}\right]\left|\left[K_{M_{0}, k}: \mathbb{Q}\right]\right| g_{M_{0}, k} !$, so

$$
r_{h} \leq 1+\operatorname{ord}_{2}\left(g_{M_{0}, k} !\right)=1+\sum_{\mu \geq 1}\left[\frac{g_{M_{0}, k}}{2^{\mu}}\right] .
$$

Now $T_{q}$ diagonalizes on neither $S_{k}(q M, \psi)$ nor $S_{k}\left(q^{2} M, \psi\right)$ if $q$ is an exceptional prime for a single newform $h \in S_{k}\left(M_{0}, \psi\right)$ for any $\mathfrak{f}\left|M_{0}\right| M$. Therefore we get an upper bound for $r$, the number of primes $q$ for which $T_{q}$ fails to diagonalize, by summing over all such newforms. Then

$$
r \leq \sum_{\operatorname{cond}(\psi)\left|M_{0}\right| M} \operatorname{dim} S_{k}^{+}\left(M_{0}, \psi\right) \cdot r_{h} \leq \sum_{\operatorname{cond}(\psi)\left|M_{0}\right| M} \operatorname{dim} S_{k}^{+}\left(M_{0}, \psi\right)\left(1+\sum_{\mu \geq 1}\left[\frac{g_{M_{0}, k}}{2^{\mu}}\right]\right) .
$$

REMARK 1. One could obtain a more explicit, though considerably larger, upper bound. For example, $g_{M_{0}, k} \leq \operatorname{dim} S_{k}\left(\Gamma_{1}\left(M_{0}\right)\right)$ for which one could use the known dimension formulas.

We conclude this investigation of diagonalization with the following "negative" result for levels divisible by a high power of $q$.

THEOREM 3.5. Let $q$ be prime, and let $\psi$ be a Dirichlet character with conductor $\mathfrak{f}=q^{\nu} M_{0}$, with $\nu \geq 0$ and $q \nmid M_{0}$. Let $M$ be an integer with $M_{0} \mid M$ and $q \nmid M$, and let $s \geq 3$ be an integer. Except possibly for finitely many $k \geq 2$ with $\psi(-1)=(-1)^{k}$ and finitely many $q, T_{q}$ is not diagonalizable on $S_{k}\left(q^{\nu+s} M, \psi\right)$.

Proof. Let $N=q^{\nu+s} M$ and $N_{0}=q^{\nu+2} M$. For each $s \geq 3$, we have $\mathfrak{f}\left|N_{0}\right| N$. Further, $q\left|N / N_{0}, q^{2}\right| N_{0}$ and $\mathfrak{f} \mid N_{0} / q$. Hence if $S_{k}(N, \psi)$ contains a newform $h$ of level $N_{0}$, then $q \notin \mathfrak{Q}_{N_{0}, h}$, so $T_{q}^{N}$ is not diagonalizable. But by Theorem 6.1 , for all but finitely many $k \geq 2$ with $\psi(-1)=(-1)^{k}$ and finitely many $q, \operatorname{dim} S_{k}^{+}\left(N_{0}, \psi\right) \geq 1$, and hence $T_{q}$ is not diagonalizable on $S_{k}(N, \psi)$.

\section{Simultaneous Hecke Eigenforms}

We now turn to the question of characterizing simultaneous Hecke eigenforms in $S_{k}(N, \psi)$ for all Hecke operators $T_{\ell}^{N}, \ell$ a prime. From the previous section and the theory of newforms, for a given simultaneous eigenform $f \in S_{k}(N, \psi)$, the only primes which need careful analysis are primes $q \mid N / N_{0}$ where $N_{0}$ is the level of the associated newform. We make this explicit.

TheOREm 4.1. Let $f \in S_{k}(N, \psi)$ be a nonzero simultaneous eigenform for all the Hecke operators $T_{\ell}^{N}$, $\ell$ a prime, and put $f \mid T_{q}^{N}=\kappa_{q} f$ for each prime $q \mid N$. Associated to $f$ is a newform $h \in S_{k}\left(N_{0}, \psi\right)$ (with $\left.\operatorname{cond}(\psi)\left|N_{0}\right| N\right)$ such that $f=\sum_{d \mid N / N_{0}} \alpha_{d} h \mid B_{d}$. Then $\alpha_{1} \neq 0$, 
and normalizing with $\alpha_{1}=1$, we have that $\alpha_{d}=\prod_{q \mid d} \alpha_{q^{\mu_{q}}}$, where $\mu_{q}=\operatorname{ord}_{q}(d)$. Further, we have $\alpha_{q^{e}}=0$ for $e \geq 3$, and

$$
\alpha_{q}=\left(\kappa_{q}-\lambda_{q}\right) \text { and } \alpha_{q^{2}}= \begin{cases}0 & q \mid N_{0}, \\ 0 & q \nmid N_{0}, \kappa_{q} \neq 0, \\ \psi(q) q^{k-1} & q \nmid N_{0}, \kappa_{q}=0,\end{cases}
$$

Proof. This is immediate from Theorem 2.1, which also indicates the possible eigenvalues $\kappa_{q}$.

REMARK 2. The converse to the above theorem is also true. Starting with a newform $h$, and choosing the $\kappa_{q}$ and $\alpha_{d}$ as in the theorem, Proposition 2.2 guarantees that $f=\sum_{d \mid M} \alpha_{d} h \mid B_{d}$ is a simultaneous eigenform for all $T_{q}^{N}$ with $q \mid N$, and hence for all $T_{\ell}^{N}, \ell$ a prime.

Now we wish to count the number of linearly independent simultaneous Hecke eigenforms that are associated to a given newform.

TheOREM 4.2. Let $h \in S_{k}\left(N_{0}, \psi\right)$ be a newform and let $N$ be an integer such that $N_{0} \mid N$. For all primes $q \mid N$, put $h \mid T_{q}^{N_{0}}=\lambda_{q} h$. The number of linearly independent simultaneous eigenforms $f \in S_{k}(N, \psi)$ which are eigenforms for all $\left\{T_{\ell}^{N}\right\}$, $\ell$ a prime and which have the same eigenvalues as $h$ under all $T_{p}, p \nmid N$ is $2^{|A|} 3^{|B|}$, where $A$ and $B$ are sets of primes dividing $N / N_{0}$ satisfying

$$
q \in B=B\left(N, N_{0}, h\right) \Longleftrightarrow q \nmid N_{0}, q^{2} \mid N / N_{0}, \lambda_{q}^{2} \neq 4 \psi(q) q^{k-1},
$$

and

$$
q \in A=A\left(N, N_{0}, h\right) \Longleftrightarrow\left\{\begin{array}{l}
q \mid N_{0} \text { and } \lambda_{q} \neq 0, \text { or } \\
q \nmid N_{0}, q \| N / N_{0}, \lambda_{q}^{2} \neq 4 \psi(q) q^{k-1}, \text { or } \\
q \nmid N_{0}, q^{2} \mid N / N_{0}, \lambda_{q}^{2}=4 \psi(q) q^{k-1}
\end{array}\right.
$$

REMARK 3. By Theorem 3 of $[\mathbf{3}]$, the first condition stated to define $A\left(q \mid N_{0}\right.$ and $\left.\lambda_{q} \neq 0\right)$ is equivalent to $q \| N_{0}$, or $q^{2} \mid N_{0}$ and $\operatorname{ord}_{q}(\operatorname{cond}(\psi))=\operatorname{ord}_{q}\left(N_{0}\right)$.

Proof. Theorem 4.1 indicates the shape of every simultaneous eigenform $f$ of level $N$ associated to the newform $h: f=\sum_{d \mid N / N_{0}} \alpha_{d} h \mid B_{d}$, where without loss, $\alpha_{1}=1$, and $\alpha_{d}$ is completely determined as the product of $\alpha_{q}$ e where $e=\operatorname{ord}_{q}(d)$. We see all such values $\alpha_{q^{e}}$ are uniquely determined except for the value of $\alpha_{q}=\kappa_{q}-\lambda_{q}$ which has as many distinct values as distinct eigenvalues $\kappa_{q}$. It is now a simple matter using Theorem 2.1 to verify that the sets $A$ and $B$ characterize those cases in which $\kappa_{q}$ can have 2 or 3 distinct eigenvalues.

\section{Examples}

Theorem 4.2 tells how to compute the number of simultaneous eigenforms in $S_{k}(N, \psi)$ associated to a newform $h \in S_{k}\left(N_{0}, \psi\right)$ with $h \mid T_{q}^{N_{0}}=\lambda_{q} h$ in terms of the sets $A$ and $B$. Knowledge of the eigenvalue $\lambda_{q}$ for $q \nmid N_{0}$ can often be problematic, but there are cases in 
which it is easy to calculate explicitly the sets $A$ and $B$. We characterize one particularly useful situation, and give some examples.

Let $N_{0} \mid N$ with $N$ and $N_{0}$ having exactly the same prime divisors. Then $B=B\left(N, N_{0}, h\right)=$ $\emptyset$, and

$$
A=A\left(N, N_{0}, h\right)=\left\{q \mid N / N_{0}: \operatorname{ord}_{q}\left(N_{0}\right)=1 \text { or } \operatorname{ord}_{q}(\operatorname{cond}(\psi))\right\} .
$$

EXAmPle 1. Let $\psi$ be a character with square-free conductor $D$, and let $N$ be an integer with $D|N| D^{2}$. Then $S_{k}(N, \psi)$ has a basis consisting of simultaneous eigenforms for all Hecke operators.

Proof. Let $N_{0}$ be such that $D\left|N_{0}\right| N$, and consider a newform $h \in S_{k}\left(N_{0}, \psi\right)$. To compute $A$, let $q \mid N / N_{0}$ be a prime. Observe that $q \mid N / N_{0}$ implies $q \mid D$. Since $N \mid D^{2}, 1 \leq \operatorname{ord}_{q}\left(N_{0}\right) \leq$ $\operatorname{ord}_{q}(N) \leq 2$, and if $\operatorname{ord}_{q}\left(N_{0}\right)=2$, then $q \nmid N / N_{0}$. Thus $q \mid N / N_{0} \operatorname{implies} \operatorname{ord}_{q}\left(N_{0}\right)=1$ and hence $q \in A$. Thus $2^{|A|}=\sigma_{0}\left(N / N_{0}\right)$ (since $N / N_{0}$ is square-free), where $\sigma_{0}(m)$ is the number of positive divisors of $m$. Since

$$
S_{k}(N, \psi)=\bigoplus_{D\left|N_{0}\right| N} \bigoplus_{d \mid N / N_{0}} S_{k}^{+}\left(N_{0}, \psi\right) \mid B_{d} \cong \bigoplus_{D\left|N_{0}\right| N} \sigma_{0}\left(N / N_{0}\right) S_{k}^{+}\left(N_{0}, \psi\right),
$$

with the isomorphism as modules for the Hecke algebra generated by $T_{p}^{N}$ for all primes $p \nmid N$, the result is clear. In the isomorphism we use the convention that for a space $S, m S=\bigoplus_{i=1}^{m} S$.

As a second example, we consider a situation in which the conductor of the character $\psi$ can be large.

EXAmple 2. Let $q$ be an odd prime and $\psi$ a character of conductor $q^{\nu}, \nu \geq 1$. Then $S_{k}\left(q^{\nu+\mu}, \psi\right), \mu \geq 0$, has a basis of simultaneous eigenforms for all the Hecke operators when $\mu=0,1$; for $\mu \geq 3$ it has such a basis only for finitely many $k$ and $q$.

Proof. The only issue concerns the diagonalizability of the operator $T_{q}^{N}$ where $N=q^{\nu+\mu}$. The case of $\mu=0,1$ is addressed by Theorem 3.3, while the case of $\mu \geq 3$ is addressed by Theorem 3.5.

EXAMPle 3. Somewhat complementary to the previous example, we consider the case of $S_{k}\left(q^{2}, 1\right)$ providing instances when $T_{q}^{N}$ can be diagonalized. To that end we consider normalized newforms $h$ of level $1, q$ and $q^{2}$ and whether $T_{q}^{N}$ can be diagonalized on [h]. For level $q^{2}$ the answer is affirmative from the theory of newforms. For each newform $h \in S_{k}(q, 1)$, Equation 5.1 yields $A=\{q\}$ providing the requisite two linearly independent simultaneous eigenforms, $h$ and $h-\lambda_{q} h$. Now consider $h \in S_{k}(1,1)$. When $k<12$ or $k=14, S_{k}(1,1)=0$, and there are no classes to consider. On the other hand, when $S_{k}(1,1) \neq 0$, the situation is more subtle. $T_{q}^{N}$ will diagonalize on $[h]$ provided that $\lambda_{q}^{2} \neq 4 q^{k-1}$. As an example, consider weight 12 . There are three simultaneous eigenforms in $S_{12}\left(q^{2}, 1\right)$ equivalent to $\Delta=(2 \pi)^{12} \sum_{n=1}^{\infty} \tau(n) e^{2 \pi i n \tau} \in$ $S_{12}(1,1)$ with Fourier coefficients given by the Ramanujan $\tau$-function. Choose a prime $q$ for which $\lambda_{q}^{2}=\tau^{2}(q) \neq 4 q^{11}$; note that this is true for all primes $q$ since $\tau(q) \in \mathbb{Z}$ while $2 q^{11 / 2}$ is not. 
Using Theorem 4.1, we produce three linearly independent simultaneous eigenforms $f=$

$h+\alpha_{q} h\left|B_{q}+\alpha_{q^{2}} h\right| B_{q^{2}}$ satisfying $f \mid T_{q}^{N}=\kappa_{q} f$ where $\kappa_{q}=0, \frac{1}{2}\left(\lambda_{q} \pm \sqrt{\lambda_{q}^{2}-4 \psi(q) q^{k-1}}\right)($ all distinct). Thus $T_{q}$ diagonalizes on $S_{k}\left(q^{2}, 1\right)$.

EXAMPLE 4. The results of section 2 also can provide a multiplicity-one theorem in the following narrow context. Let $N_{0} \geq 2$ be an integer, and consider any newform $h \in S_{k}\left(N_{0}^{2}, 1\right)$. Let $N=N_{0}^{2} N_{1}$ where the only primes dividing $N_{1}$ divide $N_{0}$. Let $f \in S_{k}(N, 1)$ be an simultaneous eigenform for all Hecke operators $T_{p}$ for $p \nmid N$ which is equivalent to $h$. Then $f=h$.

Proof. This is immediate from Proposition 2.2, since for each $q \mid N$, the $q$ th eigenvalue of $h\left(\lambda_{q}\right)$ is zero forcing $f=\sum \alpha_{d} h \mid B_{d}=h$.

\section{REMARK 4.}

Another point worth noting concerns the interpretation when the sets $A$ and $B$ are empty. In such a case, Theorem 4.2 implies there is a unique simultaneous eigenform $f$ in $S_{k}(N, \psi)$ associated to a newform $h \in S_{k}\left(N_{0}, \psi\right)$. It is not necessarily the case that the simultaneous eigenform is the newform $h$. For example, choose a prime $q \| N / N_{0}$ with $q \nmid N_{0}$. Then $B=\emptyset$ and $q \notin A$ means that $\lambda_{q}^{2}=4 \psi(q) q^{k-1} \neq 0$ and hence by Theorem $4.1, f=\sum_{d \mid N / N_{0}} \alpha_{d} h \mid B_{d}$ with $\alpha_{q}=-\lambda_{q} / 2 \neq 0$.

\section{Dimensions of spaces of newforms}

To justify the last part of Theorem 3.3, we compute a lower bound for the dimension of the space of newforms $S_{k}^{+}\left(q^{\nu+r}, \psi\right)$ where $q$ is a prime, $r \geq 2$, and $\psi$ a character with conductor $q^{\nu}, \nu \geq 0$. We make implicit use of the trace formula for Hecke operators as given in [5], in particular Ross's formula for the dimension of the space of cusp forms. For trivial character, one can find a formula for the dimension of the space of newforms in [4].

THEOREM 6.1. Let $q$ be a prime, and $\psi$ be a Dirichlet character of conductor $\mathfrak{f}=q^{\nu} M_{0}$, $q \nmid M_{0}, \nu \geq 0$. Let $r$ be an integer, $r \geq 2$, and let $M$ be any integer divisible by $M_{0}(q \nmid M)$; we further require that $\operatorname{ord}_{2}\left(M / M_{0}\right) \neq 1$. Then except for finitely many values of $k \geq 2$ with $\psi(-1)=(-1)^{k}$ and finitely many values of $q$, we have the dimension of the space of newforms $S_{k}^{+}\left(q^{\nu+r} M, \psi\right)$ is positive.

Proof. We consider the Hecke algebra generated by all operators $T_{p}$ with $p \nmid q M$, and recall our shorthand of writing $m S$ for $\bigoplus_{i=1}^{m} S$ in any isomorphism of modules for the Hecke algebra. Let $N=q^{\nu+r} M$. Then 


$$
\begin{aligned}
S_{k}(N, \psi) & =\bigoplus_{\mathfrak{f}\left|N_{0}\right| N} \bigoplus_{d \mid N / N_{0}} S_{k}^{+}\left(N_{0}, \psi\right) \mid B_{d} \\
& \cong \bigoplus_{\mathfrak{f}\left|N_{0}\right| N} \sigma_{0}\left(N / N_{0}\right) S_{k}^{+}\left(N_{0}, \psi\right) \\
& =\bigoplus_{d \mid N / \mathfrak{f}} \sigma_{0}(N / d \mathfrak{f}) S_{k}^{+}(d \mathfrak{f}, \psi) .
\end{aligned}
$$

We adapt the notation of Martin [4], who gives a formula for the dimension of the space of newforms with trivial character, and put

$$
g_{0}(d)=\operatorname{dim} S_{k}(d \mathfrak{f}, \psi), \quad g_{0}^{+}(d)=\operatorname{dim} S_{k}^{+}(d \mathfrak{f}, \psi)
$$

The above decomposition yields the following relations of dimensions:

$$
g_{0}(N / \mathfrak{f})=\sum_{d \mid N / \mathfrak{f}} g_{0}^{+}(d) \sigma_{0}(N / d \mathfrak{f})=\left(g_{0}^{+} * \sigma_{0}\right)(N / \mathfrak{f})
$$

where ${ }^{*}$ is the standard Dirichlet convolution of arithmetic functions. While $g_{0}^{+}(n)$ is not a multiplicative function, $\sigma_{0}(n)$ is, and in complete analogy with [4], we let $\lambda$ be the Dirichlet inverse of $\sigma_{0}: \lambda=\mu * \mu$ ( $\mu$ the Mobius function). Thus $\lambda$ is a multiplicative function with values (for $p$ a prime) $\lambda(p)=-2, \lambda\left(p^{2}\right)=1$, and $\lambda\left(p^{j}\right)=0$ for $j \geq 3$. Taking the Dirichlet convolution of both sides of Equation 6.1 yields

$$
\operatorname{dim} S_{k}^{+}(N, \psi)=g_{0}^{+}(N / \mathfrak{f})=\sum_{d \mid N / \mathfrak{f}} g_{0}(d) \lambda(N / d \mathfrak{f})=\left(g_{0} * \lambda\right)(N / \mathfrak{f})
$$

The goal is to use the above expression to produce a formula for the dimension of the space of newforms as a function of the prime $q$ and weight $k$. Using a parametrized version of the notation from [5], we obtain a formula for the dimension of the space of cusp forms with arbitrary character:

$$
g_{0}(d)=-s_{0}(d \mathfrak{f})-s_{1}(d \mathfrak{f})+\delta+m(d \mathfrak{f})-p(d \mathfrak{f})
$$

where letting $\omega(n)$ be the number of prime divisors of an integer $n$, we have

$$
\begin{aligned}
\left|s_{0}(d \mathfrak{f})\right| & \leq 2^{\omega(d \mathfrak{f})-2}, \\
\left|s_{1}(d \mathfrak{f})\right| & \leq \frac{1}{3} 2^{\omega(d \mathfrak{f})}, \\
\delta & = \begin{cases}1 & \text { if } k=2 \text { and } \psi=1, \\
0 & \text { otherwise, }\end{cases} \\
m(d \mathfrak{f}) & =\frac{(k-1)}{12} d \mathfrak{f} \prod_{\ell \mid d \mathfrak{f}}(1+1 / \ell)(\ell \text { a prime }), \text { and } \\
p(d \mathfrak{f}) & =\frac{1}{2} \prod_{\ell \mid d \mathfrak{f}} \operatorname{par}(\ell)(\ell \text { a prime }),
\end{aligned}
$$

where $\operatorname{par}(\ell)$ are the parabolic terms as computed in Theorem 1 of [5]. 
Now we distribute the convolution of $\lambda$ through the summands defining $g_{0}$ and obtain

$$
\begin{aligned}
& g_{0}^{+}(N / \mathfrak{f})=\sum_{d \mid N / \mathfrak{f}} g_{0}(d) \lambda(N / d \mathfrak{f}) \\
& =-\sum_{d \mid N / \mathfrak{f}} s_{0}(d \mathfrak{f}) \lambda(N / d \mathfrak{f})-\sum_{d \mid N / \mathfrak{f}} s_{1}(d \mathfrak{f}) \lambda(N / d \mathfrak{f})+\delta \sum_{d \mid N / \mathfrak{f}} \lambda(N / d \mathfrak{f}) \\
& +\sum_{d \mid N / \mathfrak{f}} m(d \mathfrak{f}) \lambda(N / d \mathfrak{f})-\sum_{d \mid N / \mathfrak{f}} p(d \mathfrak{f}) \lambda(N / d \mathfrak{f}) .
\end{aligned}
$$

If we put $N / \mathfrak{f}=q^{r} M_{1}$ with $q \nmid M_{1}$, then since $r \geq 2$,

$$
\sum_{d \mid N / \mathfrak{f}} \lambda(N / d \mathfrak{f})=\sum_{j=0}^{r} \lambda\left(q^{j}\right) \sum_{d_{1} \mid M_{1}} \lambda\left(M_{1} / d_{1}\right)=0,
$$

SO

$$
\begin{aligned}
g_{0}^{+}(N / \mathfrak{f}) & =\sum_{d \mid N / \mathfrak{f}} g_{0}(d) \lambda(N / d \mathfrak{f}) \\
& =-\sum_{d \mid N / \mathfrak{f}}\left(s_{0}(d \mathfrak{f})+s_{1}(d \mathfrak{f})\right) \lambda(N / d \mathfrak{f})+\sum_{d \mid N / \mathfrak{f}}(m(d \mathfrak{f})-p(d \mathfrak{f})) \lambda(N / d \mathfrak{f}),
\end{aligned}
$$

hence

$$
\begin{aligned}
g_{0}^{+}(N / \mathfrak{f}) & =\left|g_{0}^{+}(N / \mathfrak{f})\right| \geq \\
& \left|\sum_{d \mid N / \mathfrak{f}}(m(d \mathfrak{f})-p(d \mathfrak{f})) \lambda(N / d \mathfrak{f})\right|-\left|\sum_{d \mid N / \mathfrak{f}}\left(s_{0}(d \mathfrak{f})+s_{1}(d \mathfrak{f})\right) \lambda(N / d \mathfrak{f})\right| .
\end{aligned}
$$

We shall show that the second term is bounded and the first goes to infinity as $q$ or $k$ do which will establish our result.

We consider the second term:

$$
\left|\sum_{d \mid N / \mathfrak{f}}\left(s_{0}(d \mathfrak{f})+s_{1}(d \mathfrak{f})\right) \lambda(N / d \mathfrak{f})\right| \leq \sum_{d \mid N / \mathfrak{f}}\left(\left|s_{0}(d \mathfrak{f})\right|+\left|s_{1}(d \mathfrak{f})\right|\right)|\lambda(N / d \mathfrak{f})| \leq \sum_{d \mid N / \mathfrak{f}} 2^{\omega(d \mathfrak{f})}|\lambda(N / d \mathfrak{f})|,
$$

since from above we have that

$$
\left|s_{0}(d \mathfrak{f})\right|+\left|s_{1}(d \mathfrak{f})\right| \leq 2^{\omega(d \mathfrak{f})-2}+\frac{1}{3} 2^{\omega(d \mathfrak{f})}<2^{\omega(d \mathfrak{f})} .
$$

Writing $N / \mathfrak{f}=q^{r} M_{1}$ with $q \nmid M_{1}$ and recalling that $\mathfrak{f}=q^{\nu} M_{0}$, we have

$$
\begin{aligned}
\sum_{d \mid N / \mathfrak{f}} 2^{\omega(d \mathfrak{f})}|\lambda(N / d \mathfrak{f})| & =\sum_{j=0}^{r}\left|\lambda\left(q^{r-j}\right)\right| \sum_{d_{1} \mid M_{1}} 2^{\omega\left(q^{j+\nu} d_{1} M_{0}\right)}\left|\lambda\left(M_{1} / d_{1}\right)\right| \\
& \leq \sum_{j=0}^{r}\left|\lambda\left(q^{r-j}\right)\right| \sum_{d_{1} \mid M_{1}} 2^{1+\omega(M))}\left|\lambda\left(M_{1} / d_{1}\right)\right| \\
& \leq 2^{3+\omega(M)} \sum_{d_{1} \mid M_{1}}\left|\lambda\left(M_{1} / d_{1}\right)\right|,
\end{aligned}
$$

which is a constant depending only on $M$ and independent from $k$ and $q$. Thus it remains only to show that

$$
\left|\sum_{d \mid N / \mathfrak{f}}(m(d \mathfrak{f})-p(d \mathfrak{f})) \lambda(N / d \mathfrak{f})\right| \rightarrow \infty
$$


as $q$ or $k$ go to infinity. What we show is that

$$
\sum_{d \mid N / \mathfrak{f}}(m(d \mathfrak{f})-p(d \mathfrak{f})) \lambda(N / d \mathfrak{f})=\frac{k-1}{12} A \mathcal{M}(q)-B \mathcal{P}(q)
$$

with constants $A, B$ depending only on $M, A>0$, and $\mathcal{M}, \mathcal{P}$ functions of $q$ with the expression having the desired limits as $q$ or $k$ go to infinity.

We first consider the "Mass" term: $\sum_{d \mid N / \mathfrak{f}} m(d \mathfrak{f}) \lambda(N / d \mathfrak{f})$. For an integer $n$, let $m_{0}(n)=$ $n \prod_{\ell \mid n}(1+1 / \ell)$ where the product is over all primes $\ell$ dividing $n$. Then $m(n)=\frac{k-1}{12} m_{0}(n)$, and $m_{0}$ is a multiplicative function. Thus once again writing $N / \mathfrak{f}=q^{r} M_{1}$ with $q \nmid M_{1}$ and recalling that $\mathfrak{f}=q^{\nu} M_{0}$,

$$
\begin{aligned}
\sum_{d \mid N / \mathfrak{f}} m(d \mathfrak{f}) \lambda(N / d \mathfrak{f}) & =\frac{k-1}{12} \sum_{j=0}^{r} m_{0}\left(q^{\nu+j}\right) \lambda\left(q^{r-j}\right) \sum_{d_{1} \mid M_{1}} m_{0}\left(d_{1} M_{0}\right) \lambda\left(M_{1} / d_{1}\right) \\
& =\frac{k-1}{12}\left(m_{0}\left(q^{\nu+r}\right)-2 m_{0}\left(q^{\nu+r-1}\right)+m_{0}\left(q^{\nu+r-2}\right)\right) \sum_{d_{1} \mid M_{1}} m_{0}\left(d_{1} M_{0}\right) \lambda\left(M_{1} / d_{1}\right) \\
& =\frac{k-1}{12} \mathcal{M}(q) \sum_{d_{1} \mid M_{1}} m_{0}\left(d_{1} M_{0}\right) \lambda\left(M_{1} / d_{1}\right)
\end{aligned}
$$

where

$$
\mathcal{M}(q)= \begin{cases}q^{\nu+r-2}\left(1+\frac{1}{q}\right)(q-1)^{2} & \text { if } \nu+r-2>0 \\ q^{2}-q-1=(q-1)^{2}+q-2 & \text { if } \nu=0, r=2\end{cases}
$$

We now wish to show that $\sum_{d_{1} \mid M_{1}} m_{0}\left(d_{1} M_{0}\right) \lambda\left(M_{1} / d_{1}\right)$ is positive. Since both $m_{0}$ and $\lambda$ are multiplicative, it suffices to show this when $M_{1}=p^{e}, M_{0}=p^{f}$ are prime powers $(e+f \geq 1)$.

$$
\begin{aligned}
& \sum_{d_{1} \mid M_{1}} m_{0}\left(d_{1} M_{0}\right) \lambda\left(M_{1} / d_{1}\right)=\sum_{j=0}^{e} m_{0}\left(p^{f+j}\right) \lambda\left(p^{e-j}\right) \\
& = \begin{cases}m_{0}\left(p^{f}\right) & \text { if } e=0 \\
-2 m_{0}\left(p^{f}\right)+m_{0}\left(p^{f+1}\right) & \text { if } e=1 \\
m_{0}\left(p^{e-2+f}\right)-2 m_{0}\left(p^{e-1+f}\right)+m_{0}\left(p^{e+f}\right) & \text { if } e \geq 2\end{cases} \\
& = \begin{cases}p^{f}+p^{f-1} & \text { if } e=0 \\
p^{f+1}-p^{f}-2 p^{f-1} & \text { if } e=1 \\
p^{2}-p-1 & \text { if } e=2, f=0 \\
p^{e+f-3}(p+1)(p-1)^{2} & \text { if } e+f \geq 3,\end{cases}
\end{aligned}
$$

where we understand $p^{-1}=0$, and this sum is trivially checked to be positive for all primes $p \geq 2$. Note the case with $e=1$ (when $p=2$ ) is precluded by the theorem's hypothesis $\operatorname{ord}_{2}\left(M / M_{0}\right)=\operatorname{ord}_{2}\left(M_{1}\right) \neq 1$.

Finally we turn to the parabolic terms: $\sum_{d \mid N / \mathfrak{f}} p(d \mathfrak{f}) \lambda(N / d \mathfrak{f})$. For an integer $n$, let $p_{0}(n)=$ $\prod_{\ell \mid n} \operatorname{par}(\ell)$ where the product is over all primes $\ell$ dividing $n$, and $\operatorname{par}(\ell)$ is defined as in Theorem 1 of $[\mathbf{5}]$. Then $p(n)=(1 / 2) p_{0}(n)$, and $p_{0}$ is a multiplicative function, $p_{0}(1)=1$. Once 
again writing $N / \mathfrak{f}=q^{r} M_{1}$ with $q \nmid M_{1}$ and recalling that $\mathfrak{f}=q^{\nu} M_{0}$,

$$
\begin{aligned}
\sum_{d \mid N / \mathfrak{f}} p(d \mathfrak{f}) \lambda(N / d \mathfrak{f}) & =\frac{1}{2} \sum_{j=0}^{r} p_{0}\left(q^{\nu+j}\right) \lambda\left(q^{r-j}\right) \sum_{d_{1} \mid M_{1}} p_{0}\left(d_{1} M_{0}\right) \lambda\left(M_{1} / d_{1}\right) \\
& =\frac{1}{2}\left(p_{0}\left(q^{\nu+r}\right)-2 p_{0}\left(q^{\nu+r-1}\right)+p_{0}\left(q^{\nu+r-2}\right)\right) \sum_{d_{1} \mid M_{1}} p_{0}\left(d_{1} M_{0}\right) \lambda\left(M_{1} / d_{1}\right) \\
& =\frac{1}{2} \mathcal{P}(q) \sum_{d_{1} \mid M_{1}} p_{0}\left(d_{1} M_{0}\right) \lambda\left(M_{1} / d_{1}\right),
\end{aligned}
$$

where $\mathcal{P}(q)=\left(p_{0}\left(q^{\nu+r}\right)-2 p_{0}\left(q^{\nu+r-1}\right)+p_{0}\left(q^{\nu+r-2}\right)\right)$.

Thus it remains only to show that

$$
\left|\frac{k-1}{12} A \mathcal{M}(q)-B \mathcal{P}(q)\right| \rightarrow \infty, \text { as } k \text { or } q \rightarrow \infty
$$

with constants $A, B$ depending only on $M=M_{0} M_{1}, A>0$. The case for $k \rightarrow \infty$ is clear, so we focus on this expression as a function of $q$.

To compute $p_{0}\left(q^{\nu+j}\right)$, we set a bit of notation. Let $\mu_{j}=\left\lfloor\frac{\nu+j}{2}\right\rfloor$. From Theorem 1 of [5], we have

$$
p_{0}\left(q^{\nu+j}\right)=\operatorname{par}(q)= \begin{cases}2 q^{j} & \text { if } \nu \geq \mu_{j}+1 \\ \left(q^{\mu_{j}}+q^{\mu_{j}-1}\right) & \text { if } \nu \leq \mu_{j}, \nu+j \text { even } \\ 2 q^{\mu_{j}} & \text { if } \nu \leq \mu_{j}, \nu+j \text { odd }\end{cases}
$$

Since we need to compute $\mathcal{P}(q)=\left(p_{0}\left(q^{\nu+r}\right)-2 p_{0}\left(q^{\nu+r-1}\right)+p_{0}\left(q^{\nu+r-2}\right)\right)$ as part of $\left|\frac{k-1}{12} A \mathcal{M}(q)-B \mathcal{P}(q)\right|$, we need to break the argument into cases.

Case 0: Special case $\nu=0, r=2$.

$\left|\frac{k-1}{12} A \mathcal{M}(q)-B \mathcal{P}(q)\right|=\left|\frac{k-1}{12} A\left(q^{2}-q-1\right)-B(q-2)\right| \rightarrow \infty$ as $q \rightarrow \infty$.

Henceforth we can assume $\nu+r \geq 3$, so $\mathcal{M}(q)=q^{\nu+r-2}\left(1+\frac{1}{q}\right)(q-1)^{2}$.

Case 1: $\nu \geq \mu_{r}+1$. Then $\nu \geq \mu_{r}+1 \geq \mu_{r-1}+1 \geq \mu_{r-2}+1$. Note that this case cannot occur unless $\nu \geq 3$. Then $\mathcal{P}(q)=2 q^{r-2}(q-1)^{2}$, so

$$
\begin{aligned}
\left|\frac{k-1}{12} A \mathcal{M}(q)-B \mathcal{P}(q)\right| & \left.=\mid \frac{k-1}{12} A q^{\nu+r-2}\left(1+\frac{1}{q}\right)(q-1)^{2}\right)-B\left(2 q^{r-2}(q-1)^{2}\right) \mid \\
& =q^{r-2}(q-1)^{2}\left[\left|\frac{k-1}{12} A q^{\nu}\left(1+\frac{1}{q}\right)-B\right|\right] \rightarrow \infty \text { as } q \rightarrow \infty .
\end{aligned}
$$

Case 2: $\nu \leq \mu_{r-2}$. Then $\nu \leq \mu_{r-2} \leq \mu_{r-1} \leq \mu_{r}$.

When $\nu+r$ is even, $\mu_{r}=(\nu+r) / 2 \geq 2$, and we have

$$
\begin{aligned}
\mathcal{P}(q) & =\left(q^{\mu_{r}}+q^{\mu_{r}-1}\right)-4 q^{\mu_{r-1}}+q^{\mu_{r-2}}+q^{\mu_{r-2}-1} \\
& =\left(q^{\mu_{r}}+q^{\mu_{r}-1}\right)-4 q^{\mu_{r}-1}+q^{\mu_{r}-1}+q^{\mu_{r}-2} \\
& =q^{\mu_{r}-2}(q-1)^{2}
\end{aligned}
$$

so

$$
\begin{aligned}
\left|\frac{k-1}{12} A \mathcal{M}(q)-B \mathcal{P}(q)\right| & \left.=\mid \frac{k-1}{12} A q^{\nu+r-2}\left(1+\frac{1}{q}\right)(q-1)^{2}\right)-B\left(q^{\mu_{r}-2}(q-1)^{2}\right) \mid \\
& =q^{\mu_{r}-2}(q-1)^{2}\left[\left|\frac{k-1}{12} A q^{\mu_{r}}\left(1+\frac{1}{q}\right)-B\right|\right] \rightarrow \infty \text { as } q \rightarrow \infty .
\end{aligned}
$$

When $\nu+r$ is odd, $\mu_{r}=\mu_{r-1}=\mu_{r-2}+1$, so

$$
\mathcal{P}(q)=2 q^{\mu_{r}}-2\left(q^{\mu_{r-1}}+q^{\mu_{r-1}-1}\right)+2 q^{\mu_{r-2}}=0,
$$


and

$$
\left.\left|\frac{k-1}{12} A \mathcal{M}(q)-B \mathcal{P}(q)\right|=\mid \frac{k-1}{12} A q^{\nu+r-2}\left(1+\frac{1}{q}\right)(q-1)^{2}\right) \mid \rightarrow \infty \text { as } q \rightarrow \infty .
$$

Case 3. $\mu_{r-2}<\nu \leq \mu_{r}$.

If $\nu+r$ is even, the condition translates to $\frac{\nu+r}{2}-1<\nu \leq \frac{\nu+r}{2}$, so that $\mu_{r}=\frac{\nu+r}{2}=\nu=r$, and $\mu_{r-1}=\mu_{r-2}=\mu_{r}-1$. If $\nu+r$ is odd, it translates to $\frac{\nu+r-1}{2}-1<\nu \leq \frac{\nu+r-1}{2}$, so that $\mu_{r}=\mu_{r-1}=\frac{\nu+r-1}{2}=\nu=r-1$, and $\mu_{r-2}=\mu_{r}-1$.

If $\nu+r$ is even, we have

$$
\begin{aligned}
\mathcal{P}(q) & =\left(q^{\mu_{r}}+q^{\mu_{r}-1}\right)-4 q^{r-1}+2 q^{r-2} \\
& =\left(q^{\nu}+q^{\nu-1}\right)-4 q^{\nu-1}+2 q^{\nu-2} \\
& =q^{\nu-2}\left(q^{2}-3 q+2\right)=q^{\nu-2}(q-1)(q-2),
\end{aligned}
$$

so

$$
\begin{aligned}
\left|\frac{k-1}{12} A \mathcal{M}(q)-B \mathcal{P}(q)\right| & \left.=\mid \frac{k-1}{12} A q^{\nu+r-2}\left(1+\frac{1}{q}\right)(q-1)^{2}\right)-B\left(q^{\nu-2}(q-1)(q-2)\right) \mid \\
& =q^{\nu-2}(q-1)\left[\left|\frac{k-1}{12} A q^{r}(q-1)\left(1+\frac{1}{q}\right)-B(q-2)\right|\right] \rightarrow \infty \text { as } q \rightarrow \infty .
\end{aligned}
$$

If $\nu+r$ is odd, we have

$$
\begin{aligned}
\mathcal{P}(q) & =2 q^{\mu_{r}}-2\left(q^{\mu_{r-1}}+q^{\mu_{r-2}}\right)+2 q^{r-2} \\
& =2 q^{\nu}-2\left(q^{\nu}+q^{\nu-1}\right)+2 q^{\nu-1}=0,
\end{aligned}
$$

so

$$
\left.\left|\frac{k-1}{12} A \mathcal{M}(q)-B \mathcal{P}(q)\right|=\mid \frac{k-1}{12} A q^{\nu+r-2}\left(1+\frac{1}{q}\right)(q-1)^{2}\right) \mid \rightarrow \infty \text { as } q \rightarrow \infty .
$$

\section{References}

1. A. O. L. Atkin and J. Lehner, 'Hecke operators on $\Gamma_{0}(m)$ ', Math. Ann. 185 (1970), 134-160. MR 42 \#3022

2. YoungJu Choie and Winfried Kohnen, 'Diagonalizing "bad" Hecke operators on spaces of cusp forms', Number theory, Dev. Math., vol. 15, Springer, New York, 2006, pp. 23-26. MR MR2213826 (2006m:11056)

3. Wen Ch'ing Winnie Li, Newforms and functional equations, Math. Ann. 212 (1975), 285-315. MR 51 \#5498

4. Greg Martin, 'Dimensions of the spaces of cusp forms and newforms on $\Gamma_{0}(N)$ and $\Gamma_{1}(N)$ ', J. Number Theory 112 (2005), no. 2, 298-331. MR MR2141534 (2005m:11069)

5. Shepley L. Ross, II, 'A simplified trace formula for Hecke operators for $\Gamma_{0}(N)$ ', Trans. Amer. Math. Soc. 331 (1992), no. 1, 425-447. MR MR1053115 (92g:11043)

6. Goro Shimura, 'The special values of the zeta functions associated with Hilbert modular forms', Duke Math. J. 45 (1978), no. 3, 637-679. MR MR507462 (80a:10043)

7. 'Corrections to: "The special values of the zeta functions associated with Hilbert modular forms" [Duke Math. J. 45 (1978), no. 3, 637-679; MR 80a:10043]', Duke Math. J. 48 (1981), no. 3, 697. MR MR630592 (82j:10051) 
T. Shemanske 6188 Kemeny Hall

Dartmouth College

Hanover, NH 03755, USA

S. Treneer

6188 Kemeny Hall

Dartmouth College

Hanover, NH 03755, USA

thomas.r.shemanske@dartmouth.edu stephanie.a.treneer@dartmouth.edu
L. Walling

Department of Mathematics

University Walk Bristol, BS8 1TW,UK

malhw@bristol.ac.uk 